\title{
ezRAD: A Simplified Method for Genomic Genotyping in Non- Model Organisms
}

Here, we introduce ezRAD, a novel strategy for restriction site-associated DNA (RAD) that requires little technical expertise or investment in laboratory equipment, and demonstrate its utility for ten non-model organisms across a wide taxonomic range. ezRAD differs from other RAD methods primarily through its use of standard Illumina TruSeq library preparation kits, which makes it possible for any laboratory to send out to a commercial genome analyses core facility for library preparation and next-generation sequencing with virtually no additional investment beyond the cost of the service itself. This simplification opens RADseq to any lab with the ability to extract DNA and perform a restriction digest. ezRAD also differs from others in its flexibility to use any restriction enzyme (or combination of enzymes) that cuts frequently enough to generate fragments of the desired size range, without requiring the purchase of separate adapters for each enzyme or a sonication step, which can further decrease the cost involved in choosing optimal enzymes for particular species and research questions. We apply this method across a wide taxonomic diversity of non-model organisms to demonstrate the utility and flexibility of our approach. The simplicity of ezRAD makes it particularly useful for the discovery of Single Nucleotide Polymorphisms and targeted amplicon sequencing in natural populations of non-model organisms that have been historically understudied because of lack of genomic information. 
1 Robert J. Toonen ${ }^{1,2}$, Jonathan B. Puritz ${ }^{1,3}$, Zac H. Forsman ${ }^{1}$, Jonathan L. Whitney ${ }^{1}$, Iria Fernandez-

2 Silva $^{1,4}$, Kimberly R. Andrews ${ }^{1,5}$, Christopher E. Bird ${ }^{6}$

$3{ }^{1}$ Hawai'i Institute of Marine Biology, School of Ocean \& Earth Sciences \& Technology,

4 University of Hawai' $i$ at Mānoa, Coconut Island, 46-007 Lilipuna Road, Kāne'ohe, HI 96744.

5 22Email addresses: toonen@hawaii.edu,jpuritz@gmail.com,zac@hawaii.edu,

6 jonathanlyonwhitney@gmail.com, iriafernandezsilva@gmail.com,kimandrews@gmail.com,

7 chris.bird@tamucc.edu

$8{ }^{3}$ Current Address: Department of Wildlife and Fisheries Sciences, Texas A\&M University,

9 College Station, Texas 77843-2258

$10{ }^{4}$ Current Address: Trans-disciplinary Organization for Subtropical Island Studies (TRO-SIS),

11 University of the Ryukyus, 1 Senbaru, Nishihara, Okinawa 903-0213, Japan

$12{ }^{5}$ Current Address: Durham University, School of Biological \& Biomedical Sciences, South Road, 13 Durham, DH1 3LE, UK.

$14{ }^{6}$ Texas A\&M University - Corpus Christi, Department of Life Sciences, 6300 Ocean Drive, 15 Corpus Christi, TX 78412-5802 
17 Next-generation sequencing (NGS) has provided unprecedented access to genomic information at ever-increasing speed and reduced cost (Mardis 2008). Until recently, profiling a large number of loci was only realistically possible for organisms with well-developed genomic resources, and the high cost of developing these resources has been a major impediment to studies for non-model organisms. Despite the rapid advances of sequencing technology, and dramatic reduction in cost associated with those advances, whole-genome sequencing remains a costly hurdle to undertake for marker development in non-model organisms, especially for species with large genomes. In the fields of phylogeography, phylogenetics, and population genetics, the majority of studies do not require whole-genome sequencing, but rather a spread of loci across the genome. As a result, there has been considerable interest in simple and more cost-effective approaches to using reduced representation genome sequencing, such as Restriction site Associated DNA Sequencing, or RADseq. RADseq effectively reduces genome complexity and size by resequencing only stretches of genomic DNA adjacent to restriction endonuclease sites, providing high coverage of homologous portions of the genome from multiple individuals for comparatively low cost and effort. A multitude of strategies have emerged for RAD sequencing, including the original method (Baird et al. 2008; Etter et al. 2011; Hohenlohe et al. 2010), genotype-by-sequencing, or GBS (Elshire et al. 2011; Sonah et al. 2013), 2-enzyme GBS (Poland et al. 2012), 2b-RAD (Wang et al. 2012), and ddRAD (Peterson et al. 2012). The RADseq approach provides a powerful tool for a wide range of genetic studies and is rapidly changing the field as a result (reviewed by Rowe et al. 2011).

Despite rapidly gaining popularity, the application of RADseq has been limited to primarily model or emerging model species (Baird et al. 2008; Chutimanitsakun et al. 2011; Emerson et al. 2010; Hohenlohe et al. 2010), with only a single marine invertebrate species RADseq dataset (for the model organism Nematostella vectensis) published to date (Reitzel et al. 2013). Although it is debatable what the underlying cause of this delayed application is, applying existing protocols to non-model marine invertebrates can be challenging due to a variety of unknowns such as genome size and frequency of restriction sites. Additionally, many of the existing protocols require a significant initial investment for labs focused on Sanger sequencing and microsatellite typing. Thus, we sought to develop a simplified and general approach to RADseq that requires little to no optimization and would enable access to this powerful new approach among taxonomic groups across the tree of life. Here, we outline a novel RAD strategy that uses any restriction enzyme (or combination of enzymes) which cuts frequently enough to produce fragments suitable for sequencing, and then uses the standard Illumina TruSeq library preparation with agarose gel (or SPRI-bead) size-selection to target the fragments to be sequenced. The approach is flexible and scalable, making it possible for virtually any lab to send out restriction endonuclease digested DNA for RAD sequencing with no additional investment beyond the cost of the core lab costs for library preparation and sequencing itself. This simplification opens the door to RAD sequencing

54 for any lab with the ability to extract DNA and perform a restriction digest, a very low technical

55 bar for the application of NGS. Furthermore, this method is compatible with a wide range of 56 restriction enzymes, and does not require the purchase of new adapters for each new enzyme. 
57 Therefore, ezRAD provides flexibility for optimizing the number of unique fragments to be

58 sequenced by simple modifications to the restriction enzyme and/or size selection range used.

59 Here, we report and apply ezRAD across a wide taxonomic diversity of non-model organisms to

60 demonstrate the utility of this approach. This generalized approach, using the standard Illumina

61 TruSeq library preparation kit, will allow researchers to apply RADseq technology to a wide

62 array of research questions.

63

\section{METHODS:}

\section{DNA Extraction and Quantification}

High molecular weight DNA was extracted from preserved tissue samples using a variety of methods. For Patiria miniata, Porites compressa, Porites lobata, and Stenella longirostris, the E.Z.N.A. MicroElute Genomic DNA extraction kit (Omega) was used according to the manufacturer protocol. DNA from Cryptasterina hystera, Cryptasterina pentagona, Pocillopora damicornis, and Cellana talcosa was extracted using the DNeasy tissue extraction kit (Qiagen), and DNA from Paracirrhites arcatus was extracted using a standard Phenol-Chloroform procedure with the addition of RNase. All extractions were inspected on $2 \%$ agarose gels for the presence of impurities and lower molecular weight DNA. Samples with "smear" gel patterns were subsequently purified with AmpureXP (Agentcourt) SPRI beads using a 2:1 template to bead volume ratio. Subsequently, all extractions were quantified using AccuBlue High Sensitivity fluorescence assay on a SpectraMax M2 plate reader, using the standard protocol for a 96 well assay (Application Note \#22) with the adjustment of using the AccuBlue dye excitation and emission spectra. For all libraries, $1.5 \mu \mathrm{g}$ of DNA was precipitated in 1/10 volume Sodium Acetate and two volumes $100 \%$ ethanol at $-70^{\circ} \mathrm{C}$ for 30 minutes. DNA was pelleted by 15 min of centrifugation at $12,000 \mathrm{rpm}$. Pellets were resuspended in $24 \mu \mathrm{l}$ of dI water with $0.1 \mathrm{M}$ Tris at $65^{\circ} \mathrm{C}$.

\section{Digestion}

DNA was digested simultaneously with the isoschizomers MboI and Sau3AI (NEB) to minimize any potential impacts of methylation of DNA in digesting the genomic libraries. Each digestion was performed in $50 \mu \mathrm{l}$ reactions: $5 \mu \mathrm{l}$ NEB Buffer 4, $0.5 \mu \mathrm{l} \mathrm{BSA,} 2 \mu \mathrm{l} \mathrm{MboI,} 2.5 \mu \mathrm{l} \mathrm{Sau3AI}, 18$ $\mu \mathrm{l}$ of DNA template (roughly $1.125 \mu \mathrm{g}$ ) from above, and $22 \mu \mathrm{l}$ of dI water. Digestions were incubated at $37^{\circ} \mathrm{C}$ for 3-6 hours and then cleaned using $80 \mu$ of AmpureXP beads per reaction and eluted in $20 \mu \mathrm{l}$ of water.

\section{Illumina Library Preparation}

Cleaned digestions were inserted directly into the Illumina TruSeq DNA kit following the Sample Preparation v2 Guide starting with the "Perform End Repair" step. Digestions can be inserted into any of the three available Illumina TruSeq DNA kits including the including the newest PCR-free and Nano kits. Due to the challenges of working with non-model marine invertebrates, 
93

94

95

96

97

98

99

100

101

102

103

104

105

106

107

108

109

110

111

112

113

114

115

116

117

118

119

120

121

122

123

124

125

126

127

128

129

130

many of our libraries had less than $1 \mu \mathrm{g}$ of high molecular weight DNA with which to start the library preparation, but the Nano kit was not yet available. Due to the low starting concentration, we performed an initial PCR enrichment before gel extraction, but have had better success with the Nano kit since its release, and would recommend that approach for low initial DNA concentrations. We generally followed the TruSeq protocol, but attempted to save reagents and further lower costs by performing nearly all reaction steps in $1 / 3$ of the recommended volumes (see detailed protocol - S1), although such modifications are not necessary for the protocol. In brief, digested libraries were end repaired, 3' ends were adenylated and TruSeq adapters were ligated to the digested genomic DNA sample. Libraries were then size-selected following the Illumina TruSeq protocol using a 2\% low-melt agarose gel with $1 \mathrm{X}$ TAE buffer run at 120V for 120 minutes. The 400-500bp fragments (of which $\sim 120 \mathrm{bp}$ are the ligated adapters) were cut out with a sterile scalpel blade for each individual sample and DNA was recovered using the Qiagen MinElute Gel Extraction Kit following manufacturer instructions. After gel extraction, libraries were validated by visualization on an Agilent 2100 BioAnalyzer, quantified using qPCR, and pooled (performed by the Hawai' $i$ Institute of Marine Biology EPSCoR Core sequencing facility). Pooled libraries were then sequenced as paired-end 100bp runs on the Illumina GAIIx at HIMB.

\section{Bioinformatics}

The HIMB Core facility runs a standard quality control filter and parses the Illumina reads into FASTQ files sorted by index. Beyond that, a custom bash script (S2) was used to automate read quality filtering, reference contig assembly, read mapping, SNP calling, and SNP filtering. A brief description of each step of the analyses follows below:

Raw FASTQ files were trimmed using the program TRIM GALORE! (http://www.bioinformatics.babraham.ac.uk/projects/trim_galore/) into two different read sets. The first set of reads had only adapter sequences removed and were subsequently saved for contig assembly. The second set of reads was trimmed for adapter sequences and also removed any base that had a quality score of less than 10 (90\% probability of being correct). These reads were saved for mapping.

RAINBOW (Chong et al. 2012) clustered and assembled the first set of parsed FASTQ files into a final assembly of reference contigs. RAINBOW is specifically designed to assemble contigs from RAD sequencing. In short, it first clusters reads together that are less than 4 bp apart. These clustered reads are then recursively divided into groups representing individual allele sequences. Individual allele sequences are then assembled and merged into a final set of RAD contigs.

Quality trimmed reads were then mapped to the reference contigs using BWA (Li \& Durbin 2009) with the MEM algorithm and default parameters (with the exception of altering the number of computational threads and restricting the output to only map scores of 10 and higher). SAM files

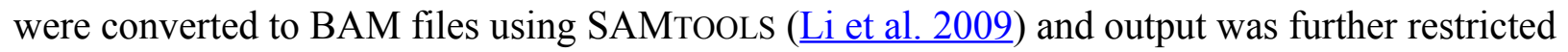
to reads with mapping quality above 15 . BAM files were then merged and realigned around 
131 INDEL regions using the mpileup command of SAMTOOLS with default parameters and the

132 additional command of outputting per-sample read depths.

133 SNP calling was performed using VARSCAN2 (Koboldt et al. 2009; Koboldt et al. 2012) using the 134 mpileup2snp command with default settings. The strand filter was removed because overlapping

135 forward and reverse reads are not expected for the insert size of this library, and the minimum

136 variant frequency was raised from $1 \%$ to $10 \%$, meaning that within one library the minimum 137 allele frequency had to be above $10 \%$ to be called a SNP. Finally, the p-value for a significant

138 variant was raised from 0.01 to 0.05 . Genotypes failing any of these filters are reported as 139 missing.

140 The raw SNP calls were then filtered using two instances of VCFtools (Danecek et al. 2011). The 141 first instance filters out INDEL loci, sites that were fixed for the minor allele, and SNPs that were 142 not genotyped in $99 \%$ of the samples. The second round of filtering removes sites with less than $14310 \mathrm{X}$ coverage and outputs the final set of SNP genotypes in VCF format.

144 Reads from the four Patiria miniata libraries were used for validation of the ezRAD technique 145 and bioinformatics pipeline. For the first test, contigs generated by RAINBOW (Chong et al. 2012) 146 for the ezRAD P. miniata libraries were aligned using BWA (Li \& Durbin 2009) with the MEM 147 algorithm and default parameters to previously published $P$. miniata genomic contigs from 148 GenBank (Assembly GCA_000285935.1). As a second test, genomic contigs were substituted 149 for the ezRAD contigs in the analysis pipeline to directly compare the number of SNPs generated 150 with different reference contigs from P. miniata.

\section{RESULTS:}

\section{Taxonomic representation}

153 We tested our generic protocol with no attempt at optimization across a range of taxonomic 154 diversity including a marine mammal (Stenella longirostris), a coral reef fish (Paracirrhites 155 arcatus), three echinoderms (Patiria miniata, Cryptasterina hystera \& $C$. pentagona), a mollusk 156 (Cellana talcosa), and three scleractinian corals (Porites compressa, P. lobata \& Pocillopora 157 damicornis). Across the range of metazoan diversity from cnidarians to vertebrates, the technique 158 worked relatively well with no modifications or attempts at optimization (Table 1).

\section{Sequencing results}

160 All attempted libraries yielded thousands to tens of thousands of variable base calls. Results 161 varied by taxon (Table 1), but as with other RAD protocols, the two factors most directly linked 162 to the number of reads per library passing quality control were initial DNA fragment sizes and 163 overall genome size, as opposed to taxonomic relatedness. When holding the size selection range 164 constant, large genome sizes and low molecular weight DNA resulted in increased numbers of 165 non-homologous DNA fragments with lesser coverage. For example, Acropora digitifera has $166 \sim 420$ megabase genome (Shinzato et al. 2011), but in combination with the suite of 
167 dinoflagellate, prokaryotic and eukaryotic symbionts inextricably associated with the coral

168 holobiont (reviewed by Ainsworth et al. 2010) this makes for an exceedingly large genomic pool

169 from which to draw fragments for reduced representation genomic sequencing. Genome size is

170 estimated from the amount of DNA (in picograms) contained in a haploid nucleus, taken from the

171 Animal Genome Size Database (http://www.genomesize.com). The corals (1115 Mb for

172 Siderastrea stellata, plus 1467 - 4694 Mb for Symbiodinium symbionts) have relatively lower

173 coverage across each contig in comparison to species with smaller genome sizes, such as the

174 limpets (421 Mb for Lottia gigantea) or the sea stars (743 Mb for Patiria miniata). The number

175 of both fragments and putative SNPs identified for P. miniata may appear high, but the species is

176 known to be extremely polymorphic - even by comparison to other sea stars in the Asterinidae

177 (Keever et al. 2009; McGovern et al. 2010; Puritz \& Toonen 2011). Further, we validate these

178 variable bases as putative SNPs against published genomic contigs (see ezRAD Validation

179 below).

180 Samples with low molecular weight DNA extractions and large genome size produced the lowest 181 quality among all libraries in our tests. Further, genomic DNA samples characterized by low 182 molecular weight fragments were also characterized by reduced adapter ligation efficiency that 183 led to a large number of sequenced fragments consisting of only adapter dimers with low quality 184 scores. For the sea star, $C$. pentagona a relatively large genome and the lowest molecular weight 185 DNA in this study resulted in the lowest quality library (Table 1). However, despite only 18.02\% 186 of the sequence reads passing QC, we still discovered over 8,000 variable base sites which is 187 more than sufficient for SNP discovery applications. Likewise, the fact that the arc-eye hawkfish 188 (Paracirrhites arcatus) has a relatively large genome (714 Mb for Cirrhitichthys aureus) together 189 with carryover of degraded DNA from the initial extraction, resulted in $P$. arcatus showing a 190 relatively low percentage of reads that passed QC (Table 1).

191 ezRAD Validation with Published Reference Genomic Contigs

192 ezRAD derived genomic sequencing reads of $P$. miniata were mapped to reference consensus 193 sequences generated from the publicly available genomic contig data from $P$. miniata on 194 GenBank. Overall, 532,467 of the 635,376 P. miniata contigs generated from the ezRAD 195 analysis pipeline mapped with high quality to publicly available genomic contigs (MAPQ mean

$196=38.66$; median 52.00; standard deviation 23.22). Approximately 15 million ezRAD reads 197 mapped with high quality to the reference, versus $\sim 21$ million reads from the publicly available 198 data set (Table 2). Most importantly, the number of variable sites, shared SNPs, and quality199 controlled SNP datasets were similar between the reference contigs from each of the two 200 approaches (Table 2).

201 SNP Discovery using Pooled and Unpooled Libraries

202 We compare results obtained by making a single library per individual for each of eight 203 individuals of the reef fish $P$. arcatus relative to two pools of four individuals each (Table 1). 204 After normalizing for lane use, we generated $2.4 \mathrm{x}$ more high quality mapped reads for the eight 205 individual libraries $\left(4.6 \times 10^{6} /\right.$ lane $)$ than for two pooled samples containing eight individuals (1.9 
206

207

208

209

210

211

212

213

214

215

216

217

x $10^{6} /$ lane) and $2 \times$ more variable base calls ( $2.6 \times 10^{5} /$ lane vs. $1.2 \times 10^{5} /$ lane). When accounting for cost however, the sample prep and sequencing was $4 \mathrm{x}$ more expensive for the 8 individuals versus two pools of four individuals, making the pooling strategy more cost effective per variable base identified. Furthermore, because of some variability among individual libraries, we identified 11.9x more shared SNPs with $>10 \mathrm{x}$ coverage between the pooled libraries $(4.35 \mathrm{x}$ $10^{4} /$ lane) than among the eight individual libraries (3.67 x 10 $3 /$ lane). For example, 975 shared SNPs were genotyped (at $>10 \mathrm{x}$ mean coverage) in all 8 individual libraries, and 635 of those (65\%) were also genotyped in both pooled libraries. By comparison, of the 3344 SNPs that were genotyped (at $>10 x$ mean coverage) between the 2 pooled libraries, only $626(19 \%)$ of those were also genotyped in all 8 individual libraries. Even without normalization for lane use, we identified more shared SNPs of higher quality for lower cost from the two pooled libraries relative to the individual libraries (Table 1; Suppl. S3).

\section{DISCUSSION:}

ezRAD, a novel approach to reduced representation genomic sequencing, differs from existing RADseq methods primarily in that it requires very little technical expertise or laboratory equipment to complete. These benefits are achieved through the use of the Illumina TruSeq library preparation kits, which also makes it possible to send digested DNA to any core lab that offers library preparation as part of their Illumina service package. This method now makes RADseq possible for any lab with the ability to perform DNA extraction and restriction digestion, an extremely low technical expertise and equipment bar to achieve NGS capability.

ezRAD is similar in concept to several other recently developed RAD methods, such as GBS, 2enzyme GBS, ddRAD and 2b-RAD (Elshire et al. 2011; Peterson et al. 2012; Poland et al. 2012; Sonah et al. 2013; Wang et al. 2012) in that we use frequent-cutting enzymes to generate fragments of the appropriate length for sequencing (usually between 300-500bp), rather than using a sonication step to sheer DNA after digestion as in the original RADseq protocol (see Table 3 for a comparison among methods). ezRAD and ddRAD both use a size selection step to eliminate inappropriately-sized fragments generated by the restriction digest. In contrast, GBS relies on a PCR step to preferentially amplify shorter fragments over longer fragments; and 2bRAD uses a special type of restriction enzyme (IIB enzymes) that cuts DNA into small, uniformly sized fragments (33-36bp) suitable for sequencing. As in GBS, ddRAD \& 2b-RAD, the number of unique fragments generated by ezRAD can be optimized by altering the restriction enzyme/s used based on the frequency of cut sites in the genome (if known). ddRAD \& ezRAD share the additional advantage that the number of fragments sequenced can be modified through the size selection step in the library preparation. However, ezRAD offers two advantages that simplify the process of choosing an appropriate restriction enzyme for a given organism and research question: 1) ezRAD generally targets just one restriction site (here both $\mathrm{MboI}$ and Sau $3 A I$ target GATC, but with different sensitivity to methylation). Assuming some knowledge of genome size and GC content, using a single cut site simplifies the calculations to predict the number of unique genomic regions within a given size range that will be generated through the 
digest. In practice any restriction enzyme, or combination of enzymes, that result in appropriately sized fragments could be used. 2) The adapters are not custom-designed for the enzyme/s used, thereby allowing researchers to try many different restriction enzymes (or combinations of enzymes) without the costly investment of new adapters for each enzyme. The ability to quickly try multiple different enzymes may be particularly beneficial for recalcitrant genomes where no prior knowledge of genome content is available, as is the case in many nonmodel organisms. Regardless of such differences among techniques, for many applications optimization of restriction enzyme and size selection range are likely irrelevant for SNP discovery, because even without any attempt at optimization, and with as few as $18 \%$ of reads passing QC, we nevertheless discovered thousands of putative SNPs in each library here (Table 1).

In order to highlight the flexibility and broad applicability of this approach, we provide an example application of this approach in which we prepared 30 libraries across a wide taxonomic range. With no adjustments to the protocol, and no attempt at optimization of any step to accommodate the taxonomic or genomic differences among the taxa, we successfully RAD sequenced a marine mammal, a fish, a mollusk, several echinoderms, and scleractinian corals. While the protocol obviously did not work equally well on all samples in the run, sequencing of all libraries yielded sufficient data for most applications from every taxon attempted (Table 1). Rather than a taxonomic bias in the success of the technique, the success of a library appears to be a direct result of the initial quality of DNA that went into the library preparation and the genome size. We find that starting with the highest possible molecular weight DNA and testing for the standard QC along the way makes the greatest difference in the amount of useable sequence data resulting from a run.

\section{Costs}

The total cost of preparing and sequencing our 30 libraries in two lanes of an Illumina GAIIx flow cell was $\$ 9,600$. We were able to reduce the cost per dataset to $\sim \$ 320$ (start to finish) by buying all reagents and constructing the libraries in our laboratory and sequencing through our HIMB core facility, making the library preparation cost on the order of $\sim \$ 60$. Current prices for library preparation at academic institutions that offer the service in the USA are on the order of \$200-300 for the TruSeq library preparation and approximately the same for $1 / 6^{\text {th }}$ of MiSeq or $1 / 12^{\text {th }}$ an Illumina GAIIx sequencing lane, making the total price of an ezRAD run on the order of $\$ 500-\$ 600$ USD if sent out. Some will note that this cost is substantially higher than the published library prep costs for other RAD methods which can be as low as $\$ 5 /$ sample, but such estimates do not include the cost of sequencing, which is the majority of the cost of RADseq. However, it is also important to note that there is a trade-off between the ultimate cost per library and the initial investment to begin the process of library development. Initial investment for most RADseq methods would include, at the minimum, acquiring a Solid Phase Reversible Immobilization (SPRI) bead kit $(\sim 1250)$, a 96-well magnet for the SPRI cleanups $(\sim \$ 620)$, restriction enzymes $(\sim 2-300)$, a high accuracy DNA quantification kit $(\sim \$ 100$, plus $\sim \$ 2000$ if an accurate fluorometer is not already available), plus the initial order of the custom oligo adapters with barcode sequences (which could also run into thousands of dollars depending on the number 
286

287

288

289

290

291

292

293

294

295

296

297

298

299

300

301

302

303

304

305

306

307

308

309

310

311

312

313

314

315

316

317

318

319

320

\section{1}

322

323

324

325

of enzymes and barcodes desired for the protocol). If all of those reagents are used up fully in several hundred library preparations, the cost per library will be quite low, on the order of $\$ 5$ $10 /$ sample, whereas the cost of sequencing ( $\$ 250$ per sample) remains fixed. However, if only a few libraries are made before those reagents expire, the cost of the reagents alone may be greater than the total price for the 30 ezRAD libraries we ran here. Even using ezRAD, labs who plan to do only a few libraries are unlikely to want to invest in the Illumina TruSeq sample library preparation kit ( $\sim 2600)$, and are much better off sending out to a commercial service for their needs at a higher price per sample, but much lower overall cost. Ultimately, there are trade-offs for every method, and for labs that plan to prepare and run many RADseq libraries, there are more cost-effective options available (Table 3); however for labs that need only a few runs for SNP discovery or marker development for targeted amplicon sequencing, and have none of the required supplies listed above in hand, ezRAD is the least technically challenging and most costeffective option currently available.

\section{Pooled versus Unpooled Libraries}

In comparing pooled and unpooled libraries, we find that SNP discovery is more cost effective in libraries constructed with DNA from pooled individuals in comparison to multiple runs of single individuals (Figure 1). For a substantial examination of pooled NGS sample strategies see Gautier et al. (2013). Here, our simple comparison of one library per individual on eight individuals of the reef fish Paracirrhites arcatus relative to two pools of four individuals each indicate pooled libraries are more cost-effective for SNP discovery (Figure 1, Suppl. S3).

Comparing our individual libraries illustrates that there is some variability among libraries in the markers recovered, likely due to imprecision in the gel size selection step; ultimately, the proportion of SNPs that were shared among all individuals drops as we compare across more of the individual libraries (Suppl. S3). Still, the highest coverage SNPs tend to be those shared among libraries, and the slope of loss asymptotes after about 5 individuals for reasonable levels of coverage (Suppl. S3). Even without normalization for lane use, we identified 3x more shared SNPs from the pooled libraries than from the individual libraries (Table 1, Suppl. S3). Although we used only eight individuals in this simple comparison of pooled and unpooled libraries, the best results for SNP discovery were in pooled libraries in which we had 20 or more individuals and other genomic resources against which to compare our variable base calls (e.g., P. miniata or C. talcosa, Table 1). Based on our findings here, our future efforts for SNP discovery would likely use two pooled libraries of many individuals $(>20)$ each as the most cost-effective strategy to identify hundreds to thousands of high quality shared SNPs or loci for targeted amplicon sequencing (e.g., Puritz et al. 2012) that could be reliably used for genotyping from these libraries (Suppl. S3).

\section{Benefits and trade-offs of ezRAD}

Ultimately, there are tradeoffs to consider for each of the various approaches to reduced representation genomic sequencing strategies (reviewed by Wang et al. 2012). Although ezRAD is simple, works without optimization across a broad diversity of metazoan taxa, and requires little initial investment beyond the direct cost of NGS, it is important to consider that this 
326

327

328

329

330

331

332

333

334

335

336

337

338

339

340

341

342

343

344

345

346

347

348

349

350

351 We are grateful to Amy Eggers of the Hawai' $i$ Institute of Marine Biology EPSCoR Evolutionary

352 Genetics Core Facility for her assistance in the preparation of the libraries and sequencing. We

353 also thank Brian Bowen, Steve Karl, Mahdi Belcaid, Dan Barshis, Matt Iacchei, Mareike Sudek,

354 and Ingrid Knapp, as well as all the other members of the ToBo lab for their discussion, advice

355 and support of this work, and putting up with us taking over the lab to try out this crazy idea. We

356 also thank Rick Grosberg and Brenda Cameron for generously allowing C. Bird to work on early

357 RADseq attempts in their laboratory. This is contribution \#\#\#\# from the Hawai' $i$ Institute of

358 Marine Biology and SOEST \#\#\#\#. 
360

361

362

363

364

365

366

367

368

369

370

371

372

373

374

375

376

377

378

379

380

381

382

383

384

385

386

387

388

389

390

391

392

393

394

395

396

397

398

399

400

Ainsworth TD, Thurber RV, and Gates RD. 2010. The future of coral reefs: a microbial perspective. Trends in Ecology \& Evolution 25:233-240.

Baird NA, Etter PD, Atwood TS, Currey MC, Shiver AL, Lewis ZA, Selker EU, Cresko WA, and Johnson EA. 2008. Rapid SNP discovery and genetic mapping using sequenced RAD markers. PLoS One 3:e3376.

Chong Z, Ruan J, and Wu C-I. 2012. Rainbow: an integrated tool for efficient clustering and assembling RAD-seq reads. Bioinformatics 28:2732-2737.

Chutimanitsakun Y, Nipper RW, Cuesta-Marcos A, Cistué L, Corey A, Filichkina T, Johnson EA, and Hayes PM. 2011. Construction and application for QTL analysis of a Restriction Site Associated DNA (RAD) linkage map in barley. Bmc Genomics 12:4.

Danecek P, Auton A, Abecasis G, Albers CA, Banks E, DePristo MA, Handsaker RE, Lunter G, Marth GT, and Sherry ST. 2011. The variant call format and VCFtools. Bioinformatics 27:2156-2158.

Elshire RJ, Glaubitz JC, Sun Q, Poland JA, Kawamoto K, Buckler ES, and Mitchell SE. 2011. A robust, simple genotyping-by-sequencing (GBS) approach for high diversity species. PLoS One 6:e19379.

Emerson KJ, Merz CR, Catchen JM, Hohenlohe PA, Cresko WA, Bradshaw WE, and Holzapfel CM. 2010. Resolving postglacial phylogeography using high-throughput sequencing. Proceedings of the National Academy of Sciences 107:16196-16200.

Etter PD, Bassham S, Hohenlohe PA, Johnson EA, and Cresko WA. 2011. SNP discovery and genotyping for evolutionary genetics using RAD sequencing. Molecular methods for evolutionary genetics: Springer, 157-178.

Hohenlohe PA, Bassham S, Etter PD, Stiffler N, Johnson EA, and Cresko WA. 2010. Population genomics of parallel adaptation in threespine stickleback using sequenced RAD tags. PLoS Genet 6:e1000862.

Keever CC, Sunday J, Puritz JB, Addison JA, Toonen RJ, Grosberg RK, and Hart MW. 2009. Discordant distribution of populations and genetic variation in a sea star with high dispersal potential. Evolution 63:3214-3227.

Koboldt DC, Chen K, Wylie T, Larson DE, McLellan MD, Mardis ER, Weinstock GM, Wilson RK, and Ding L. 2009. VarScan: variant detection in massively parallel sequencing of individual and pooled samples. Bioinformatics 25:2283-2285.

Koboldt DC, Zhang Q, Larson DE, Shen D, McLellan MD, Lin L, Miller CA, Mardis ER, Ding L, and Wilson RK. 2012. VarScan 2: somatic mutation and copy number alteration discovery in cancer by exome sequencing. Genome Research 22:568-576.

Li H, and Durbin R. 2009. Fast and accurate short read alignment with Burrows-Wheeler transform. Bioinformatics 25:1754-1760.

Li H, Handsaker B, Wysoker A, Fennell T, Ruan J, Homer N, Marth G, Abecasis G, and Durbin R. 2009. The sequence alignment/map format and SAMtools. Bioinformatics 25:20782079.

Mardis ER. 2008. The impact of next-generation sequencing technology on genetics. Trends in Genetics 24:133. 
401

402

403

404

405

406

407

408

409

410

411

412

413

414

415

416

417

418

419

420

421

422

423

424

425

426

427
McGovern TM, Keever CC, Saski CA, Hart MW, and Marko PB. 2010. Divergence genetics analysis reveals historical population genetic processes leading to contrasting phylogeographic patterns in co-distributed species. Molecular Ecology 19:5043-5060.

Peterson BK, Weber JN, Kay EH, Fisher HS, and Hoekstra HE. 2012. Double digest RADseq: an inexpensive method for de novo SNP discovery and genotyping in model and non-model species. PLoS One 7:e37135.

Poland JA, Brown PJ, Sorrells ME, and Jannink J-L. 2012. Development of high-density genetic maps for barley and wheat using a novel two-enzyme genotyping-by-sequencing approach. PLoS One 7:e32253.

Puritz JB, Addison JA, and Toonen RJ. 2012. Next-generation phylogeography: a targeted approach for multilocus sequencing of non-model organisms. PLoS One 7:e34241.

Puritz JB, and Toonen RJ. 2011. Coastal pollution limits pelagic larval dispersal. Nature Communications 2:226.

Reitzel A, Herrera S, Layden M, Martindale M, and Shank T. 2013. Going where traditional markers have not gone before: utility of and promise for RAD sequencing in marine invertebrate phylogeography and population genomics. Molecular Ecology.

Rowe H, Renaut S, and Guggisberg A. 2011. RAD in the realm of next-generation sequencing technologies. Molecular Ecology 20:3499-3502.

Shinzato C, Shoguchi E, Kawashima T, Hamada M, Hisata K, Tanaka M, Fujie M, Fujiwara M, Koyanagi R, and Ikuta T. 2011. Using the Acropora digitifera genome to understand coral responses to environmental change. Nature 476:320-323.

Sonah H, Bastien M, Iquira E, Tardivel A, Légaré G, Boyle B, Normandeau É, Laroche J, Larose S, and Jean M. 2013. An improved genotyping by sequencing (GBS) approach offering increased versatility and efficiency of SNP discovery and genotyping. PLoS One 8:e54603.

Wang S, Meyer E, McKay JK, and Matz MV. 2012. 2b-RAD: a simple and flexible method for genome-wide genotyping. Nature Methods 9:808-810. 


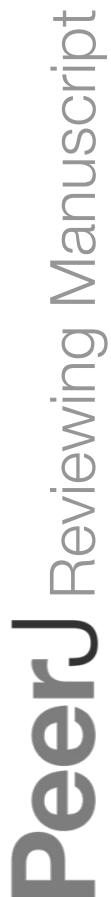

PeerJ reviewing PDF | (v2013:07:650:1:1:ACCEPTED 15 Oct 2013) 


\section{Figure 1}

Bar graph comparing pooled and unpooled libraries.

Relative proportion of high quality mapped reads, total SNPs, shared SNPs with greater than 10x coverage, and cost when employing one of two strategies: 1) preparing one library for every individual (8 individuals here), or 2) preparing two libraries of four pooled individuals. For all categories except Cost, taller bars represent better performance.

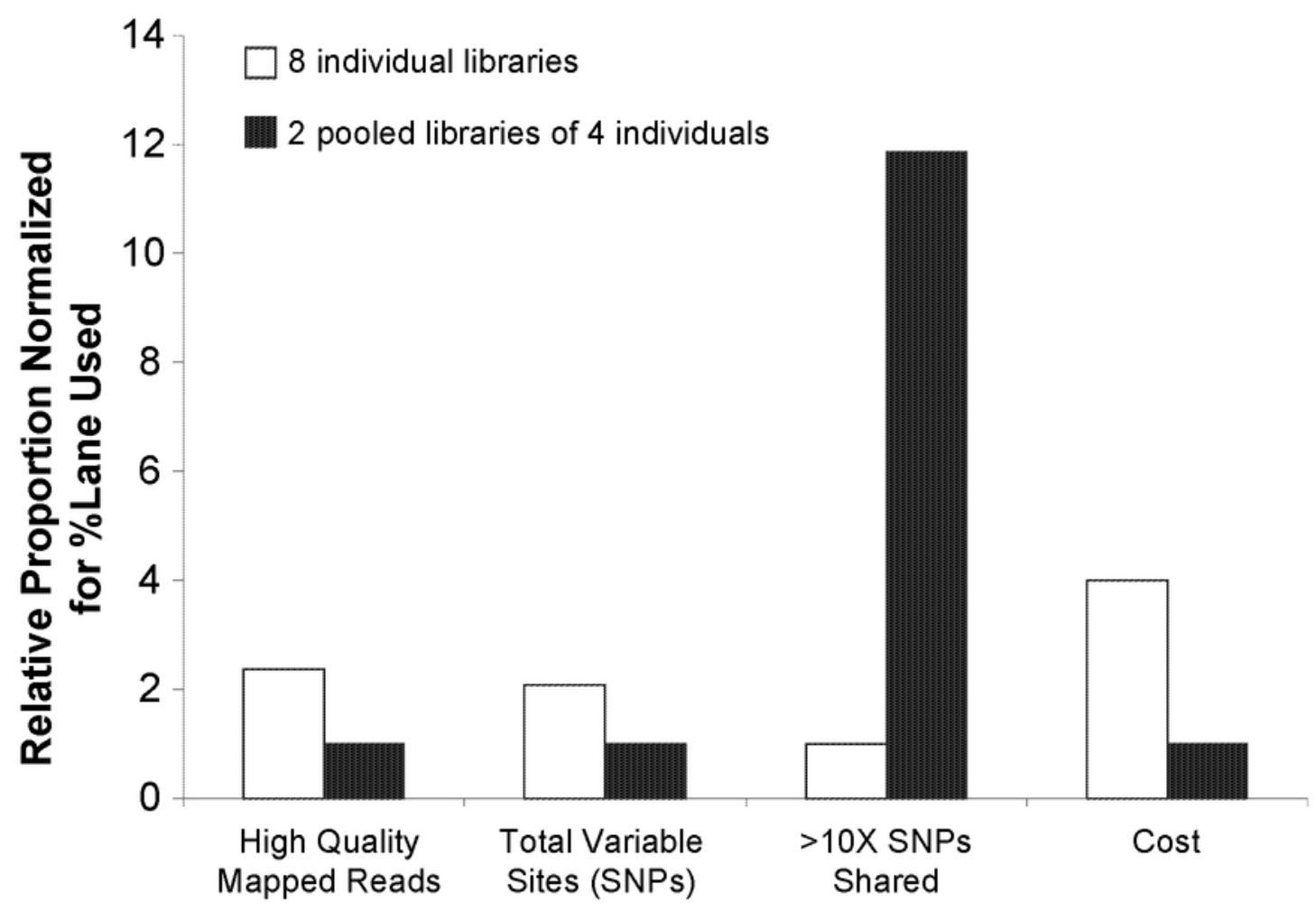

Categories 


\section{Table $\mathbf{1}_{\text {(on next page) }}$}

Summary of ezRAD results from 2 lanes of lllumina GAllx sequencing across a range of taxonomic diversity.

Organisms run with ezRAD include: the limpet Cellana talcosa; sea stars Cryptasterina hystera, C. pentagona \& Patiria miniata; reef fish Paracirrhites arcatus; corals Porites lobata, P. compressa \& Pocillopora damicornis; and the spinner dolphin Stenella longirostris. Lane use indicates the proportion of a single lane of PE100bp sequencing on the GAllx flow cell. Library prep specifies what those libraries contained. Paired reads are the number of reads in each index parsed file returned after initial quality control (QC) filter from the sequencer. Reads and \% Pass QC are the number of sequence reads remaining after excluding all sequences for which Phred scores were $<20$, contained adapter sequences, or were less than 20bp long after adapters were cut. Mapped reads and High quality mapped reads are the number of reads overall and the number reads that passed quality control, respectively, that were assembled de novo into contigs. Contig statistics and polymorphic SNP counts reported here come from the bash script pipeline described in Supplement S2. 


\begin{tabular}{|c|c|c|c|c|c|c|c|c|c|c|c|c|c|}
\hline Species & $\begin{array}{l}\text { Lane } \\
\text { Use }\end{array}$ & $\begin{array}{c}\text { Library } \\
\text { Prep }\end{array}$ & $\begin{array}{l}\text { Paired } \\
\text { Reads }\end{array}$ & $\begin{array}{c}\text { Reads } \\
\text { Passing } \\
\text { QC }\end{array}$ & $\begin{array}{c}\% \\
\text { Pass } \\
\text { QC }\end{array}$ & $\begin{array}{c}\text { No. } \\
\text { Contig } \\
\text { s }\end{array}$ & $\begin{array}{l}\text { Mapped } \\
\text { Reads }\end{array}$ & $\begin{array}{c}\text { High } \\
\text { Quality } \\
\text { Mapped }\end{array}$ & $\begin{array}{l}\text { Variable } \\
\text { Sites }\end{array}$ & $\begin{array}{c}\text { Shared } \\
\text { SNPs }\end{array}$ & $\begin{array}{c}>10 X \\
\text { SNPs } \\
\text { shared }\end{array}$ & $\begin{array}{c}>30 X \\
\text { SNPs } \\
\text { shared }\end{array}$ & $\begin{array}{c}\text { SNPs } \\
\text { Per } \\
\text { Contig }\end{array}$ \\
\hline $\begin{array}{l}\text { Cellana } \\
\text { talcosa }\end{array}$ & $1 / 3$ & $\begin{array}{l}2 \text { peols of } \\
24 \text { mdivid }\end{array}$ & $8,109,327$ & $4,472,365$ & 55.15 & 189,444 & $8,389,669$ & $3,483,833$ & 127,609 & 73,014 & 49,761 & 9,997 & 0.26 \\
\hline $\begin{array}{c}\text { Cryptasterina } \\
\text { hystera }\end{array}$ & $1 / 12$ & $\begin{array}{l}1 \text { peol of } \\
10 \text { individ }\end{array}$ & $6,441,832$ & $3,880,080$ & 60.23 & 27,007 & $2,242,589$ & 655,509 & 36,666 & 36,666 & 31,127 & 12,827 & 1.15 \\
\hline $\begin{array}{c}\text { Cryptasterina } \\
\text { pentagona }\end{array}$ & $1 / 12$ & $\begin{array}{l}1 \text { pool of } \\
10 \text { individ }\end{array}$ & $1,804,165$ & 325,137 & 18.02 & 4,354 & $1,452,171$ & 148,580 & 9,501 & 9,501 & 8,058 & 3,538 & 1.85 \\
\hline $\begin{array}{l}\text { Patiria } \\
\text { miniata }\end{array}$ & $3 / 4$ & $\begin{array}{l}4 \text { pools of } \\
20 \text { ivdivid }\end{array}$ & $\begin{array}{c}15,632,98 \\
2 \\
\end{array}$ & $\begin{array}{c}13,132,26 \\
7\end{array}$ & 84.00 & 635,376 & $\begin{array}{c}47,718,93 \\
1 \\
\end{array}$ & $\begin{array}{c}14,987,37 \\
2 \\
\end{array}$ & $1,167,981$ & 187,597 & $\begin{array}{c}143,25 \\
4\end{array}$ & 21,914 & 0.23 \\
\hline $\begin{array}{c}\text { Paracirrhites } \\
\text { arcatus }\end{array}$ & $2 / 3$ & $\begin{array}{l}8 \text { tagged } \\
\text { individ }\end{array}$ & $\begin{array}{c}13,586,62 \\
5 \\
\end{array}$ & $5,261,386$ & 38.72 & 205,360 & $\begin{array}{c}22,892,81 \\
7 \\
\end{array}$ & $3,088,806$ & 171,712 & 2,705 & 2,447 & 366 & 0.01 \\
\hline $\begin{array}{c}\text { Paracirrhites } \\
\text { arcatus }\end{array}$ & $1 / 6$ & $\begin{array}{l}2 \text { pools of } \\
4 \text { inglivid }\end{array}$ & $2,733,965$ & 995,543 & 36.41 & 13,340 & $2,980,976$ & 324,331 & 20,512 & 10,221 & 7,249 & 2,082 & 0.54 \\
\hline $\begin{array}{c}\text { Pocillopora } \\
\text { damicornis }\end{array}$ & $1 / 6$ & $\begin{array}{l}2 \text { nged } \\
\text { nd vid }\end{array}$ & $3,289,007$ & $2,929,932$ & 89.08 & 164,553 & $5,710,298$ & $2,956,701$ & 232,734 & 110,454 & 80,221 & 21,658 & 0.49 \\
\hline $\begin{array}{c}\text { Porites } \\
\text { compressa }\end{array}$ & $1 / 4$ & $\begin{array}{l}3 \text { ta sged } \\
\text { In divid }\end{array}$ & $5,512,212$ & $3,852,694$ & 69.89 & 123,874 & $7,011,801$ & $2,338,160$ & 297,648 & 77,346 & 60,149 & 13,040 & 0.49 \\
\hline $\begin{array}{c}\text { Porites } \\
\text { lobata }\end{array}$ & $1 / 4$ & $\begin{array}{l}\text { Jagged } \\
\text { individ }\end{array}$ & $7,131,427$ & $3,926,250$ & 55.06 & 95,887 & $7,587,534$ & $2,689,310$ & 275,769 & 65,731 & 47,617 & 9,419 & 0.50 \\
\hline $\begin{array}{c}\text { Stenella } \\
\text { longirostris }\end{array}$ & $1 / 3$ & $\begin{array}{c}4 \text { pools of } \\
2 \text { individ }\end{array}$ & $7,660,053$ & $2,563,007$ & 33.46 & 43,427 & $9,910,871$ & $1,022,661$ & 69,208 & 7,502 & 7,280 & 3,828 & 0.17 \\
\hline
\end{tabular}




\section{Table 2 (on next page)}

Validation of ezRAD data against genomic contigs.

Comparison of ezRAD results using two different sets of reference contigs, the original ezRAD analysis pipeline contigs and published genomic contigs for the seastar Patiria miniata. 


\begin{tabular}{|c|c|c|}
\hline Reference Type & ezRAD & Genomic Contigs \\
\hline Number of Contigs & 635,376 & 179,756 \\
\hline Mapped Reads & $47,718,931$ & $26,130,869$ \\
\hline High Quality Mapped & $14,987,372$ & $21,997,385$ \\
\hline Variable Sites & $1,167,981$ & $1,156,633$ \\
\hline Shared SNPs & 187,597 & 151,742 \\
\hline$>10 X$ Shared SNPs & 143,254 & 114,620 \\
\hline
\end{tabular}




\section{Table 3 (on next page)}

Comparison of most commonly used RAD sequencing methodologies and associated costs. 


\begin{tabular}{|c|c|c|c|c|c|c|c|c|}
\hline & $\begin{array}{c}\text { No. of } \\
\text { enzyme } \frac{\sigma}{\frac{\rho}{\mathrm{s}}}\end{array}$ & $\begin{array}{c}\text { Cut } \\
\text { frequency }\end{array}$ & $\begin{array}{l}\text { Shearing } \\
\text { required }\end{array}$ & $\begin{array}{c}\text { Size } \\
\text { selection }\end{array}$ & $\begin{array}{c}\text { Library prep } \\
\text { time \& } \\
\text { required } \\
\text { expertise }\end{array}$ & $\begin{array}{c}\text { Initial } \\
\text { outlay cost }\end{array}$ & $\begin{array}{l}\text { Subsequent } \\
\text { library cost per } \\
\text { sample }\end{array}$ & $\begin{array}{c}\text { Scalability to } \\
\text { reduce } \\
\text { overall cost } \\
\text { per sample }\end{array}$ \\
\hline ezRAD & 1 or more & Frequent & No & Yes & Low & Very Low & Moderate & Low \\
\hline RAD tags & $1 \sum$ & Rare & Yes & Yes & High & High & Low & Low \\
\hline GBS & $1 \stackrel{\frac{c}{3}}{\frac{d}{d}}$ & $\begin{array}{l}\text { Rare or } \\
\text { frequent }\end{array}$ & No & No & Moderate & High & $\begin{array}{l}\text { Moderate to } \\
\text { Very Low }\end{array}$ & Low \\
\hline $\begin{array}{l}\text { 2-enzyme } \\
\text { GBS }\end{array}$ & $2 \stackrel{0}{\square}$ & $\begin{array}{l}\text { Rare }+ \\
\text { frequent }\end{array}$ & No & No & Moderate & High & $\begin{array}{l}\text { Moderate to } \\
\text { Very Low }\end{array}$ & Low \\
\hline ddRAD & 2 (1) & Frequent & No & Yes & Moderate & High & Very Low & Moderate \\
\hline 2b-RAD & 10 & Frequent & No & No & Moderate & High & Low & Moderate \\
\hline
\end{tabular}




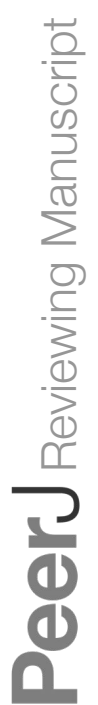

